\title{
Structure Defines Function: Clinically Relevant Mutations in ErbB Kinases
}

Janina Niggenaber, ${ }^{\ddagger}$ Julia Hardick, ${ }^{\ddagger}$ Jonas Lategahn, ${ }^{*}$ and Daniel Rauh*

Faculty of Chemistry and Chemical Biology, TU Dortmund University, Otto-Hahn-Strasse 4a, 44227 Dortmund (Germany) and Drug Discovery Hub Dortmund (DDHD) am Zentrum für Integrierte Wirkstoffforschung (ZIW), 44227 Dortmund (Germany).

$\neq$ J.N. and J.H. contributed equally.

*eMail: jonas.lategahn@tu-dortmund.de (J.L.), daniel.rauh@tu-dortmund.de (D.R.). Phone: +49 (0)231/7557080. Twitter: @DDHDortmund. Web: DDHDortmund.de. 

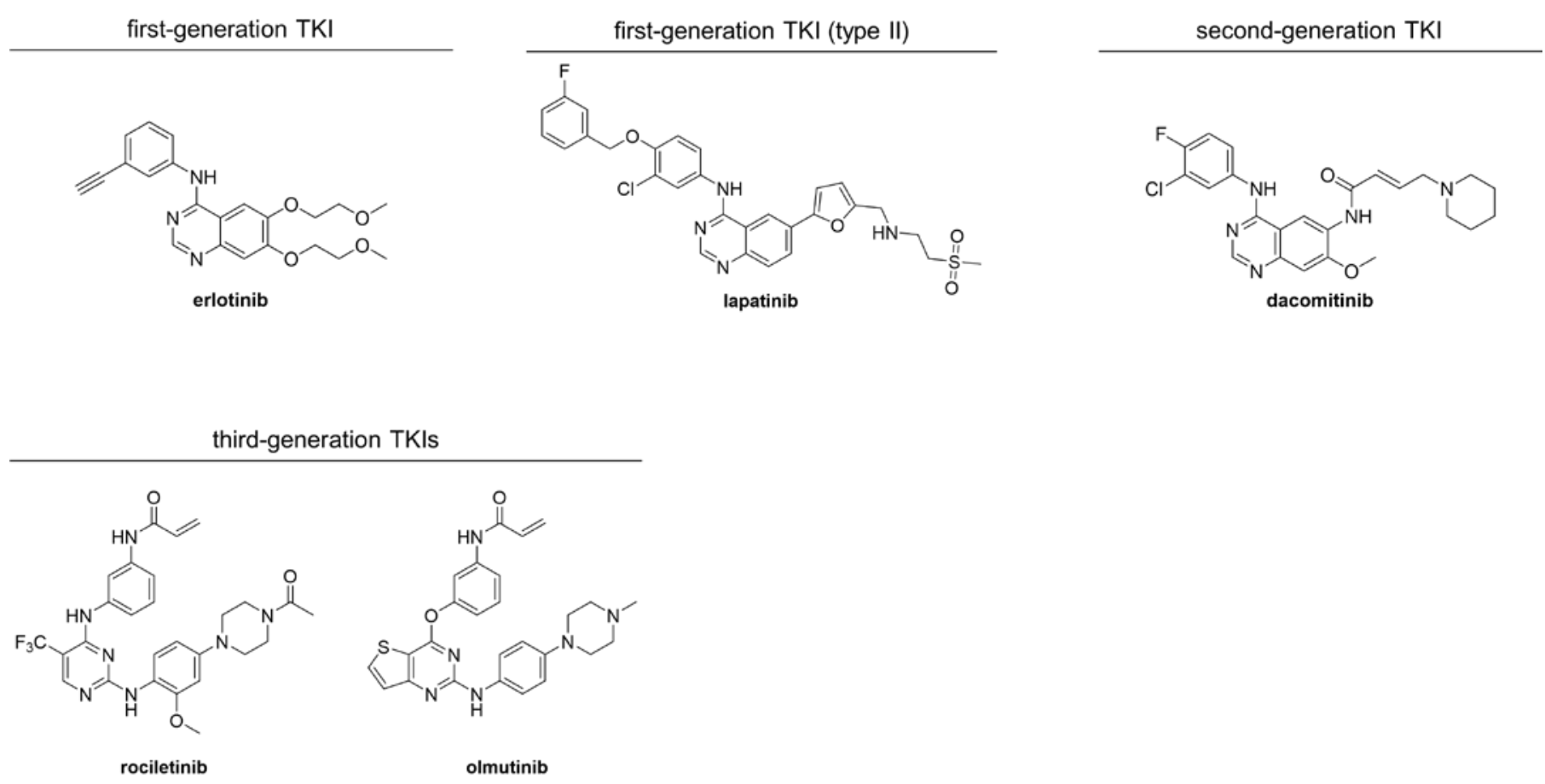

Figure S1. Chemical structures of representative examples of the three TKI-generations of EGFR. 